\title{
Learning curve for uniportal video-assisted thoracoscopic surgery lobectomy-results from 120 consecutive patients
}

\author{
Xiaochuan Liu ${ }^{1 \#}$, Xiaosang Chen ${ }^{2 \#}$, Yaxing Shen ${ }^{2}$, Hao Wang ${ }^{2}$, Mingxiang Feng ${ }^{2}$, Lijie Tan ${ }^{2}$, Thomas A. \\ D'Amico ${ }^{3}$
}

${ }^{1}$ Department of Thoracic Surgery, Guangan People's Hospital, Guangan 638000, China; ${ }^{2}$ Department of Thoracic Surgery, Zhongshan Hospital of Fudan University, Shanghai 200032, China; ${ }^{3}$ Department of Cardiothoracic Surgery, Duke University Medical Center, Durham, NC, USA

Contributions: (I) Conception and design: M Feng, TA D’Amico; (II) Administrative support: L Tan; (III) Provision of study materials or patients: X Liu, X Chen; (IV) Collection and assembly of data: Y Shen, H Wang; (V) Data analysis and interpretation: Y Shen; (VI) Manuscript writing: All authors; (VII) Final approval of manuscript: All authors.

\#These authors contributed equally to the manuscript.

Correspondence to: Mingxiang Feng, MD. Department of Thoracic Surgery, Zhongshan Hospital of Fudan University, 180 Fenglin Road, Shanghai 200032, China. Email: Feng.mingxiang@zs-hospital.sh.cn; Thomas A. D’Amico, MD. Duke University Medical Center, Box 3496, Duke South, White Zone, Room 3589, Durham, NC 27710, USA. Email: thomas.damico@duke.edu.

Background: Uniportal video-assisted thoracoscopic surgery (VATS) is considered a technically demanding procedure. The learning curve, which directly influences the adoption of uniportal VATS, has not been described. In this study, we aimed to describe the learning curve for uniportal VATS lobectomy from our single center's experience.

Methods: Uniportal VATS lobectomy was started in October 2013 in Zhongshan Hospital, Fudan University. Since then, a total of 120 consecutive patients who underwent uniportal VATS lobectomy were retrospectively enrolled. Surgical videos were reviewed to determine the operation time, to which cumulative sum (CUMSUM) method was applied to evaluate the learning phases of the procedure. Accordingly, patients' clinical features in different phases were collected and compared to determine the learning curve for uniportal VATS lobectomy.

Results: Among the 120 consecutive patients reviewed from October 2013 to September 2014, the CUMSUM curve showed its inflection at patient number 44: the first 30 patients were in the ascending phase, the second 30 patients were in the plateau phase, and the remaining patients were in the descending phase. Comparable CUMSUM results were recorded both from upper and not-upper lobectomy. Intraoperatively, more repeated stapler attempts $(73 \%$ versus $13 \%$ and $5 \%, \mathrm{P}<0.001)$ and higher conversion rate ( $17 \%$ versus $7 \%$ and $2 \%, \mathrm{P}=0.028)$ were recorded in ascending phase $v$ s. the plateau phase and descending phase, respectively. Post-operatively, the morbidity, mortality and the length of hospital stay were similar before and after the learning curve cases.

Conclusions: In a center with conventional VATS experience, the learning period of uniportal VATS lobectomy was characterized by repeated stapler attempts, and the volume requirements would be approximately 30 cases to reach the performance plateau. Upper lobectomy seemed not more difficult to learn than lower or middle lobectomy in uniportal VATS.

Keywords: Uniport; video-assisted thoracoscopic surgery (VATS); lobectomy; learning curve

Submitted Jul 05, 2018. Accepted for publication Aug 15, 2018.

doi: $10.21037 /$ jtd.2018.08.87

View this article at: http://dx.doi.org/10.21037/jtd.2018.08.87 


\section{Introduction}

The learning curve for surgery was termed to describe the case volume that a surgeon required to reach the plateau of performance $(1,2)$. To date, although uniportal videoassisted thoracoscopic surgery (VATS) has been welcomed in a number of medical centers (3-5), the technique has not been adopted in most centers, presumably due to intrinsic difficulty in learning the procedure.

In previous publications, the learning curve for VATS has been only vaguely described, ranging from 15 to 200 cases, and the learning curve was attributed to a variety of factors (6). Since there would be more vessels to be dissected, VATS upper lobectomy was considered of higher difficulty than VATS lower lobe resection (7). In contrast, the learning curve for VATS uniportal lobectomy has not been as well described (8).

Based on our primary results from uniportal VATS lobectomy (9), we herein reported our experience in 120 consecutive cases and described the learning curve for uniportal VATS lobectomy.

\section{Methods}

The study was approved by the institutional review board and the ethics committee of Zhongshan Hospital, Fudan University. Written informed consent was obtained from all patients. The uniportal VATS lobectomy was indicated to both malignant and benign lesions for the surgical resection of one target lung. The surgeries were performed by the same senior consultant surgeon (Lijie Tan) from Zhongshan Hospital, Fudan University, and the first uniportal VATS lobectomy was started in October 2013 (10). Clinical features including age, sex, forced expiratory volume in the first second $\left(\mathrm{FEV}_{1}\right)$, lesion location and diameter, histology, operation duration, morbidity and mortality were recorded.

\section{Surgical technique of uniportal VATS lobectomy}

\section{Anesthesia and analgesia}

All patients received a combination of epidural and general anesthesia, and were provided with patient-controlled analgesia (PCA) postoperatively. After intravenous induction, patient was intubated with a double-lumen endotracheal tube to accomplish single lung ventilation. Patients' vital signs were monitored during the operation. All patients would be extubated at the end of surgery and transferred to the ward.

\section{Position}

The patient was kept in a folding knife gesture (with the cranial side raised up and caudal side pushed down) in lateral decubitus position. Intra-operatively, the surgeon and the assistant stood at the abdominal side of the patient. The assistant surgeon stood at the footstool, and helped holding the thoracoscope (Karl Storz, GmbH \&Co. Tuttlingen, Germany). The entire procedure was performed under the screen observation and the surgical video was recorded.

\section{Uniportal VATS lobectomy}

In uniportal VATS lobectomy, a 2.5 -cm single incision was made at $4^{\text {th }}$ intercostal space along the anterior axillary line for upper or middle lobe resection and $5^{\text {th }}$ intercostal space for inferior lobe resection. A soft plastic wound protector was applied to the incision without rib-spreading procedure. During the procedure, the $10-\mathrm{mm} 30$ degree thoracoscope was kept in the superior side of the incision. The harmonic shear (Ethicon Endo-Surgery Inc., Cincinnati, OH, USA) or hook electro-cautery were applied to accomplish the surgical resection of a target lobe through the single incision.

The bronchus, vein and artery were divided anatomically, and dissected separately using endoscopic staplers (Covidien, Mansfield, MA, USA or Ethicon Endo-Surgery Inc., Cincinnati, OH, USA) or ligated by using hem-o-locks (Weck Surgical, Teleflex, Limerick, USA) before dissection. The dissection order varied as per the fissure development and the tumor location: Usually, in the cases of lower or middle lobe lesions, the dissection order was vein, artery and bronchus. In the cases of upper lobe lesions, the dissection order would be switched to artery, vein and bronchus (Figure 1).

The specimen was removed using a specimen bag (Ethicon Endo-Surgery Inc., Cincinnati, OH, USA). Systematic mediastinal lymph node dissection was performed after the removal of the target lobe. A 24-Fr chest tube was inserted at the end of the operation, and the chest tube would be removed in the cases there was no air leakage and the volume of drainage was less than $200 \mathrm{~mL}$ per day 24 hours post-operatively.

\section{Analysis}

Clinical information from all included patients was collected from the institution's database by trained surgical coordinators. All data collected was tabulated using Microsoft Excel for further analysis. Surgical videos were reviewed independently by experienced surgeons 


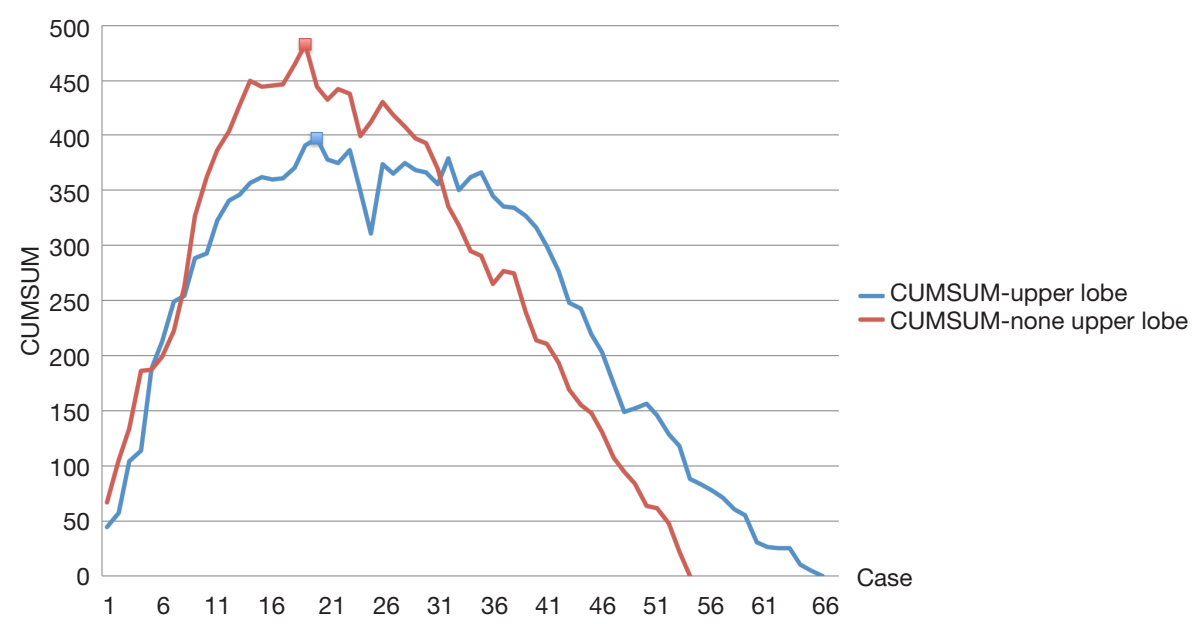

Figure 1 The CUMSUM plot for upper and none-upper uniportal VATS lobectomy. The CUMSUM curve showed that the inflection point was at patient number 20 in upper lobectomy and patient number 19 in none-upper lobectomy. VATS, video-assisted thoracoscopic surgery.

(Mingxiang Feng, Lijie Tan). The operation duration was recorded as the skin-to-skin time for the entire procedure, and the duration of lobectomy and lymphadenectomy was recorded separately. The repeated stapler attempt across the mediastinal vessels was recorded when the stapler failed to pass through the mediastinal vessels by more than 3 times on the same target vessel. Data analysis was undertaken using SPSS software (SPSS, Inc., Chicago, IL, version 17.0). Variables were compared using the Student's $t$-test, Mann-Whitney $\mathrm{U}$ test and Chi-square test. A twosided $\mathrm{P}$ value of less than 0.05 was considered statistically significant.

The CUSUM method was used for quantitative assessment of the learning curve $(11,12)$, which is the running total of differences between the individual data points and the mean of all data points. The CUSUM for the variable of interest in the first patient was the difference between the value for the first patient and the mean for all patients. The CUSUM for the second patient was the previous patient's CUSUM added to the difference obtained for the second patient. This recursive process continued until the CUSUM for the last patient was calculated as zero. The clinical results including the tumor characteristics, operative findings, post-operative morbidity and mortality were compared between the time periods within and after learning curve periods.

\section{Results}

Between October 2013 and September 2014, a total of 120 consecutive uniportal VATS lobectomies were performed in Zhongshan Hospital of Fudan University. By reviewing the surgical videos, 8 conversions $(6.7 \%)$ were observed. Additional port or ports were introduced in 4 cases $(3.3 \%)$ : 2 cases due to the failure for stapler pass across the superior pulmonary vein and 2 cases due to the iatrogenic injury to the pulmonary artery. Thoracotomy was performed in 4 conversions $(3.3 \%)$ for pulmonary artery bleeding ( 2 cases), difficult lymph nodes (1 case) and severe adhesions in thoracic cavity (1 case). The total operation duration, together with the duration of lobectomy, are shown in Figure 2.

\section{CUMSUM analysis}

The CUMSUM of lobectomy duration was plotted in chronological case order. Among the 120 consecutive patients, the CUMSUM value inflected at patient number 44. Patients 1-30 constituted the ascending slope of the curve (Phase 1), patients 31-60 constituted the plateau part of the curve (Phase 2), and patients 61-120 constituted the descending slope (Phase 3). The CUNSUM curve of uniportal VATS lobectomy is shown in Figure 3.

There were 66 cases of upper lobectomy (55\%) in the study cohort. The CUMSUM curves of upper and nonupper lobectomy are plotted separately: The inflection point was at patient number 20 in upper lobectomy and patient number 19 in non-upper lobectomy. The CUMSUM curves were similar between upper and non-upper uniportal VATS lobectomy (shown in Figure 1). 


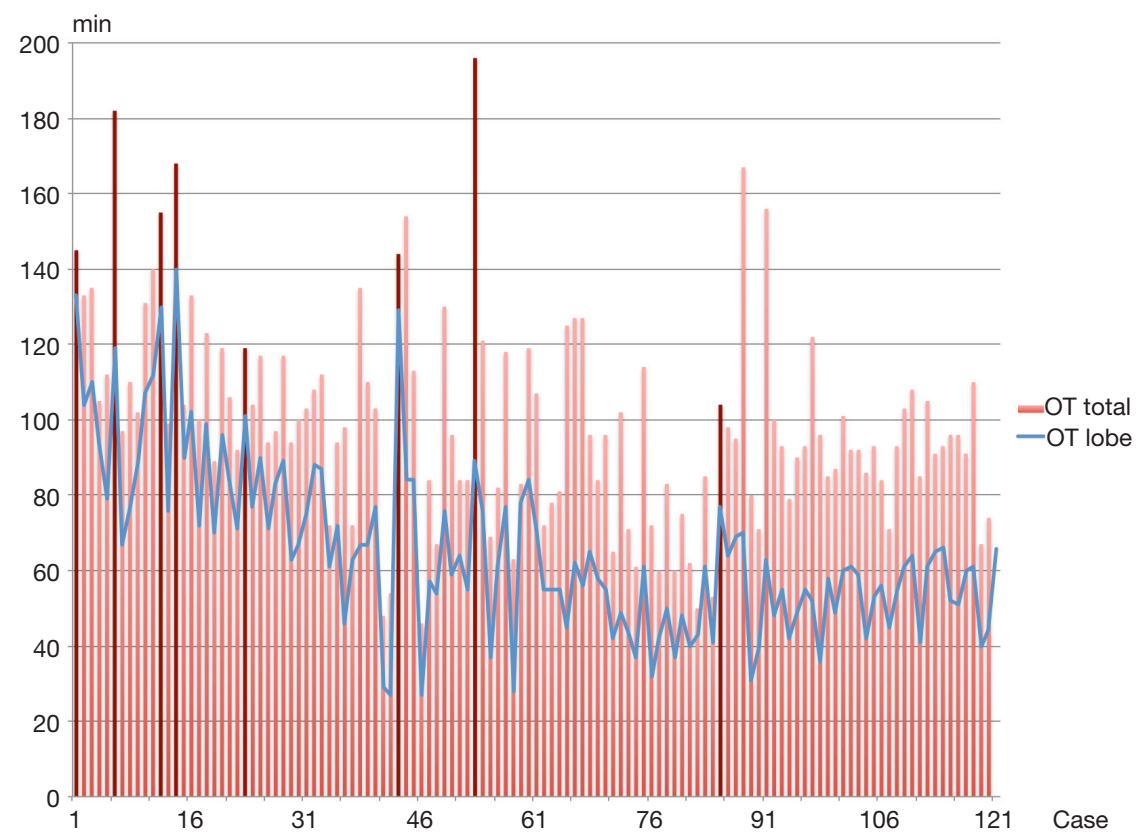

Figure 2 The time cost for uniportal VATS lobectomy. The total operation time (OT total) was shown in red column, and the operation time for lobectomy (OT lobe) was shown in blue curve. The conversion cases were marked in brown column. VATS, video-assisted thoracoscopic surgery.

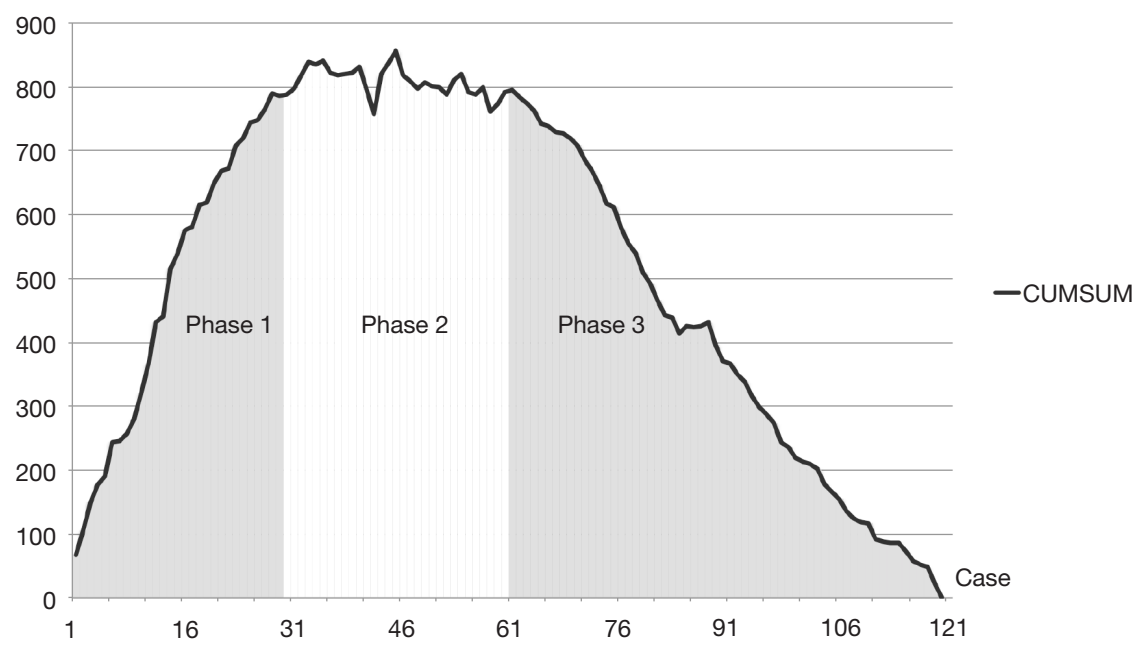

Figure 3 The CUMSUM plot for uniportal VATS lobectomy. The CUMSUM curve for the duration of lobectomy (OT lobe): The number 1-30 patients consisted the ascending slope of the curve (Phase 1), the number 31-60 patients were at the plateau part of the curve (Phase 2), and the 61-120 patients consisted the descending slope (Phase 3). VATS, video-assisted thoracoscopic surgery.

\section{Clinical features}

The clinical features were comparable, including age, sex, $\mathrm{FEV}_{1}$, lesion location/diameter, less-developed fissure, and thoracic adhesion rate among the 3 phases. Decreasing operation duration $(117.4 \pm 23.3,98.7 \pm 33.1$, and
$90.9 \pm 20.0 \mathrm{~min}$ in Phase $1,2,3$, respectively, $\mathrm{P}<0.001$ ) and decreasing lobectomy duration $(92.1 \pm 20.7,65.9 \pm 22.5$, and 52.6 $\pm 10.4 \mathrm{~min}$ in Phase 1, 2, 3, respectively, $\mathrm{P}<0.001$ ) were observed. In Phase 1, significantly higher conversion rate (17\%) was observed than in Phase $2(7 \%)$ and Phase $3(2 \%)$. 
Table 1 Clinical features

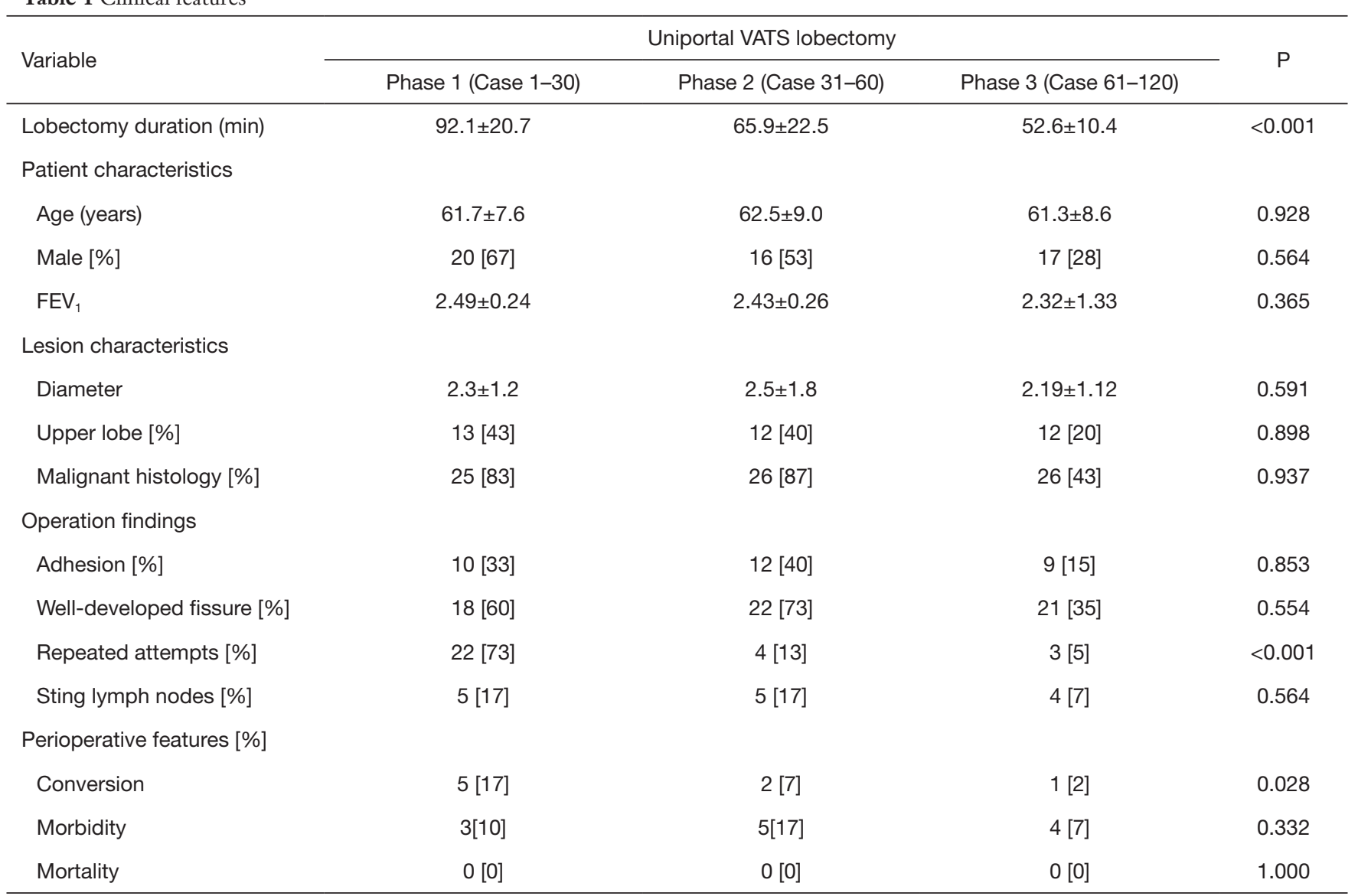

VATS, video-assisted thoracoscopic surgery; $\mathrm{FEV}_{1}$, forced expiratory volume in the first second.

Repeated attempts during mediastinal vessel dissection were more commonly recorded in Phase 1, than Phase 2 and Phase 3 (Table 1).

A total of 12 patients developed complications, including 8 with prolonged air leakages lasting more than 5 days, 3 with atelectasis requiring bronchoscopic suction after the surgery and 1 with empyema required prolonged chest tube drainage. The complication rates were not differed among the 3 phases of uniportal VATS lobectomy. No mortality occurred in any of the 3 phases.

\section{Discussion}

In this study, we analyzed the overall and procedurespecific operative times for uniportal VATS lobectomy and described the learning curve of this demanding procedure. The results from our single center suggested that a relatively short period was required to learn uniportal VATS lobectomy among experienced surgeons, and that similar cases volume would be required to reach the plateau of performance for both upper and non-upper lobectomies.

The learning curve of for multi-port VATS has been described as variable and vague in previous publications $(13,14)$. To minimize the potential bias to the study, the surgical videos of the uniportal VATS lobectomy were reviewed to determine the procedure-specific duration, which was recorded from the beginning of hilum dissection until removal of the specimen, excluding the time for thoracoscopic adhesiolysis and mediastinal lymph node dissection, which was unpredictable and varied significantly from procedure to procedure (15).

The relatively short learning curve may be explained by the following reasons. Firstly, the surgeons in our center have extensive experience in minimally invasive surgery, and this previous experience likely facilitated the 
conversion from multi-port to uniportal VATS lobectomy, particularly with the regard to familiarity with the thoracoscopic appearance of pulmonary anatomy (16-18). Secondly, the surgeons were trained in the uniportal technique prior to initiating this study by a surgeon with extensive uniportal VATS lobectomy experience. Thirdly, our center uses dedicated thoracoscopic instruments, which may be more important in the learning curve for uniportal VATS lobectomy than for multiport VATS lobectomy (19) despite the ergonomic advantages of direct visualization (20).

Generally, the surgery could be divided into two parts: The part that was within the surgical plan $(\mathrm{Pi})$ and the part that was out of the surgical plan (Po), and the learning of the surgery could be considered as the procedure that increase rate of the $\mathrm{Pi} / \mathrm{Po}$. The Po part was usually consisted of the errors made during the surgery. In the previous publications by Tong and associates, the errors made during the surgery could be divided into technical and congenital subtypes (21). Meanwhile, the repeated attempts during hilum dissection, as a technical error, were more commonly recorded before reaching the learning curve plateau. The congenital error, however, was not so common before and after the learning curve: In this study, the surgeon was experienced in conventional VATS, which made them familiar with the anatomical structures, and the congenital errors did not occur as much as the novice surgeons did described in the previous publications (22). Meanwhile, the difficulty on these experienced hands was how to pass the stapler across the hilum vessels in uniportal set. The angle between the incision and the mediastinal interspace required accumulated experience of manipulation, which explained the repeated attempts (technical errors) symbolic at the beginning of uniportal VATS.

The study further explored the learning curve differences between uniportal upper and lower lobectomy: While the average time cost was longer in upper lobectomy, the case volume required to reach the learning curve plateau was nearly identical between upper and lower lobectomy. In conventional VATS lobectomy, mastering the upper lobe dissection was technically more demanding than lower lobectomy. However, in uniportal VATS, the learning of upper lobectomy was not more difficult than lower lobe resection. The modified dissection order of mediastinal vessel was one of the important contributors to the relatively short learning curve for uniportal upper lobectomy: Firstly, the incision was located at $4^{\text {th }}$ intercostal space in uniportal VATS upper lobectomy, which is close to the Marshall space between the truncus anterior and the upper border of pulmonary vein, and the pulmonary artery was easily identified and dissected through the incision $(23,24)$. Secondly, the vein was taken following the artery in uniportal VATS upper lobectomy: The switched dissection order would minimize the iatrogenic injury to the truncus anterior branch, and decreased repeated attempts when passing the staplers across the superior pulmonary vein, therefore decreased the Po part and resulted in short learning curve in upper lobectomy (25).

In this cohort, a significant lower incidence of conversion and pulmonary artery bleeding was recorded after the ascending phase of the learning curve, as would be expected. In this study, the operation duration was significantly decreased after ascending phase of the learning curve, without increased morbidity or mortality in the cohort. The use of vessel loop on the pulmonary artery facilitated arterial repair when needed, and therefore shortened the operative duration (increased $\mathrm{Pi} / \mathrm{Po}$ ), with minimized conversions for additional ports.

In this study, we conclude that the learning curve for VATS uniportal lobectomy was relatively short in experienced hands, and upper lobe/non-upper lobe dissection shared similar case volume requirements to reach the performance plateau. The primary limitations of this study include its retrospective design and an inability to accurately characterize an individual surgeon's previous experience. It is possible that the learning curve as described in this study would vary in other centers with either more or less experience. In addition, we emphasize the importance of adequate exposure and training to uniportal VATS lobectomy prior to clinical practice. As was described in a previous publication, a randomized and controlled trial that would address all questions on uniportal VATS is likely not feasible (26). However, analysis of the experience from other centers may be useful in understanding the safety, feasibility and efficacy of uniportal lobectomy, as compared to multiport VATS lobectomy (27).

\section{Acknowledgements}

Supported by the American Association for Thoracic Surgery's Evarts A. Graham Memorial Traveling Fellowship.

Funding: This work supported by the National Natural Science Foundation of China (Grant No. 81400681 and No. 81370587). 


\section{Footnote}

Conflicts of Interest: The authors have no conflicts of interest to declare.

Ethical Statement: The study was approved by the institutional review board and the Ethics Committee of Zhongshan Hospital, Fudan University (approval No. B2017-042). Written informed consent was obtained from all patients.

\section{References}

1. Cao C, Petersen RH, Yan TD. Learning curve for videoassisted thoracoscopic lobectomy. J Thorac Cardiovasc Surg 2014;147:1727.

2. Petersen RH, Hansen HJ. Learning curve associated with VATS lobectomy. Ann Cardiothorac Surg 2012;1:47-50.

3. Rocco G, Martucci N, La Manna C, et al. Ten-year experience on 644 patients undergoing single-port (uniportal) video-assisted thoracoscopic surgery. Ann Thorac Surg 2013;96:434-8.

4. Gonzalez-Rivas D, Paradela M, Fernandez R, et al. Uniportal video-assisted thoracoscopic lobectomy: two years of experience. Ann Thorac Surg 2013;95:426-32.

5. Kara HV, Balderson SS, D'Amico TA. Modified uniportal video-assisted thoracoscopic lobectomy: Duke approach. Ann Thorac Surg 2014;98:2239-41.

6. Li X, Wang J, Ferguson MK. Competence versus mastery: the time course for developing proficiency in videoassisted thoracoscopic lobectomy. J Thorac Cardiovasc Surg 2014;147:1150-4.

7. McKenna RJ, Houck W, Fuller CB. Video-assisted thoracic surgery lobectomy: experience with 1,100 cases. Ann Thorac Surg 2006;81:421-5; discussion 425-6.

8. Liu CC, Shih CS, Pennarun N, et al. Transition from a multiport technique to a single-port technique for lung cancer surgery: is lymph node dissection inferior using the single-port technique? Eur J Cardiothorac Surg 2016;49:i64-i72.

9. Shen Y, Wang H, Feng M, et al. Single- versus multipleport thoracoscopic lobectomy for lung cancer: a propensity-matched study. Eur J Cardiothorac Surg 2016;49:148-53.

10. Feng M, Shen Y, Wang H, et al. Uniportal video assisted thoracoscopic lobectomy: primary experience from an Eastern center. J Thorac Dis 2014;6:1751-6.

11. Chaput de Saintonge DM, Vere DW. Why don't doctors use cusums? Lancet 1974;1:120-1.

12. Tekkis PP, Senagore AJ, Delaney CP, et al. Analysis of the surgical learning curve using the cumulative sum (CUSUM) method. Ann Surg 2005;242:83-91.

13. Zhao H, Bu L, Yang F, et al. Video-assisted thoracoscopic surgery lobectomy for lung cancer: the learning curve. World J Surg 2010;34:2368-72.

14. Arora KS, Khan N, Abboudi H, et al. Learning curves for cardiothoracic and vascular surgical procedures--a systematic review. Postgrad Med 2015;127:202-14.

15. Li Y, Wang J. Analysis of lymph node impact on conversion of complete thoracoscopic lobectomy to open thoracotomy. Thorac Cancer 2015;6:704-8.

16. Kim HK, Choi YH. The feasibility of single-incision video-assisted thoracoscopic major pulmonary resection performed by surgeons experienced with a twoincision technique. Interact Cardiovasc Thorac Surg 2015;20:310-5.

17. Okyere S, Attia R, Toufektzian L, et al. Is the learning curve for video-assisted thoracoscopic lobectomy affected by prior experience in open lobectomy? Interact Cardiovasc Thorac Surg 2015;21:108-12.

18. Ng CS, Gonzalez-Rivas D, D'Amico TA, et al. Uniportal VATS-a new era in lung cancer surgery. J Thorac Dis 2015;7:1489-91.

19. Carrott PW Jr, Jones DR. Teaching video-assisted thoracic surgery (VATS) lobectomy. J Thorac Dis 2013;5:S207-11.

20. Bertolaccini L, Viti A, Terzi A. Ergon-trial: ergonomic evaluation of single-port access versus three-port access video-assisted thoracic surgery. Surg Endosc 2015;29:2934-40.

21. Meyerson SL, Tong BC, Balderson SS, et al. Needs assessment for an errors-based curriculum on thoracoscopic lobectomy. Ann Thorac Surg 2012;94:368-73.

22. Tong BC, Gustafson MR, Balderson SS, et al. Validation of a thoracoscopic lobectomy simulator. Eur J Cardiothorac Surg 2012;42:364-9; discussion 369.

23. Gonzalez-Rivas D, de la Torre M, Fernandez R, et al. Single-port video-assisted thoracoscopic left upper lobectomy. Interact Cardiovasc Thorac Surg 2011;13:539-41.

24. Gonzalez-Rivas D, Fieira E, Mendez L, et al. Single-port video-assisted thoracoscopic anatomic segmentectomy and right upper lobectomy. Eur J Cardiothorac Surg 2012;42:e169-71.

25. Gonzalez-Rivas D, Stupnik T, Fernandez R, et al. 
Intraoperative bleeding control by uniportal videoassisted thoracoscopic surgery. Eur J Cardiothorac Surg 2016;49:17-24.

26. Wang BY, Liu CY, Hsu PK, et al. Single-incision versus multiple-incision thoracoscopic lobectomy and

Cite this article as: Liu X, Chen X, Shen Y, Wang H, Feng M, Tan L, D'Amico TA. Learning curve for uniportal videoassisted thoracoscopic surgery lobectomy-results from 120 consecutive patients. J Thorac Dis 2018;10(8):5100-5107. doi: $10.21037 /$ jtd.2018.08.87 segmentectomy: a propensity- matched analysis. Ann Surg 2015;261:793-9.

27. Sihoe AD. The evolution of minimally invasive thoracic surgery: implications for the practice of uniportal thoracoscopic surgery. J Thorac Dis 2014;6:S604-17. 